\title{
Chemical Etching and TEM Crystalline Quality Assessment of Single Crystalline ZnSe Ingots Grown by $\mathrm{I}_{2}$ Vapor Phase Transport
}

\author{
Raúl L. D’Elía ${ }^{1}$, Myriam H. Aguirre ${ }^{2}$, Eduardo A. Heredia ${ }^{1}$, María C. Di Stefano ${ }^{3}$, Ana M. \\ Martínez ${ }^{1}$, Alfredo J.Tolley ${ }^{4,5}$, Javier L. M. Núñez García ${ }^{1,3}$, Adriano Geraci ${ }^{4}$, Edgardo \\ Cabanillas $^{6}$, Horacio R. Cánepa ${ }^{1}$ and Alicia B. Trigubó ${ }^{1,3,{ }^{*}}$ \\ ${ }^{1}$ CINSO-UNIDEF, Juan Bautista de la Salle 4397, 1603, Villa Martelli, Buenos Aires, Argentina \\ ${ }^{2}$ Dpto de Quím Inorg, Fac de Cs Quím, Univ Complutense, 28040, Madrid, Spain \\ ${ }^{3}$ FRBA-UTN, Medrano 951, 1179, C.A.B.A., Argentina \\ ${ }^{4}$ CNEA-CAB, San Carlos de Bariloche, 8402, Río Negro, Argentina \\ ${ }^{5}$ CONICET, Av. Rivadavia 1917, 1033, CABA, Argentina \\ ${ }^{6}$ UNSAM/CNEA-CAC, Av. Gral Paz 1499, 1650, San Martín, Buenos Aires, Argentina
}

\begin{abstract}
Crystalline defects were studied in single crystalline $\mathrm{ZnSe}$ grown by chemical transport using $\mathrm{I}_{2}$ as gaseous carrier. Transmission electronic microscopy determined an excellent structural order in the micrometric and nanometric range. Larger material areas were studied by chemical etching using different reagents to determine average dislocations density and average adjacent subgrains misorientation. Comparable micrographic results of different reagents are shown. Characterization values of ZnSe commercial substrate grown by High Pressure Bridgman (HPB) have been compared to those that correspond to our grown material wafers. Characterization proved that the semiconductor crystalline quality in our wafers is appropriate for optical devices.
\end{abstract}

Keywords: Single crystalline ZnSe, II-VI Semiconductors, $\left(\mathrm{I}_{2}\right)$ Chemical transport, Chemical etching, Transmission and scanning electronic microscopy.

\section{INTRODUCTION}

$\mathrm{II}-\mathrm{VI}$ semiconductors based on $\mathrm{ZnSe}$ are indispensable for the manufacture of laser diodes in the blue-green range of the spectrum due to its energy band gap $(2.715 \mathrm{eV}$ at $300 \mathrm{~K})$. In a nearby future their immediate application in optical devices will determine high quality materials demand. Different techniques were used for growing and doping epitaxial films according to requirements [1-3].

Growth conditions optimization is necessary to advance in the knowledge and comprehension of the involved mass transference processes and in the diffusive and convective effects to obtain a good quality single crystalline material $[4,5]$. The quality of detectors and optical devices prepared with this material critically depends on their properties [6]. A lattice parameter change of $0.27 \%$ and thermal expansion coefficients differences between substrate (GaAs) and epitaxy (ZnSe), determine dislocations causing interface defects [7].

"Address correspondence to this author at the CINSO-UNIDEF, Juan Bautista de la Salle 4397, 1603, Villa Martelli, Buenos Aires, Argentina;

Tel: +0541 47098100; Fax: +054147098100; E-mail atrigubo@citedef.gob.ar
Our aim was to devise a procedure to assess the quality of the crystals grown at the laboratory by the chemical vapor transport (CVT) method studying defects presence in the ZnSe grown material and comparing its characterization results with those that corresponded to the commercial window used in the laboratory whose reports indicate that was grown by HPB (High Pressure Bridgman). As no lineal defects were detected by transmission electronic microscopy (TEM) in the micrometric and nanometric range of the lab grown material, then chemical etching was employed to determine dislocations density and adjacent subgrains misorientation.

\section{EXPERIMENTAL}

Electrical and optical properties of semiconductors are highly determined by impurities so it is important to obtain its synthesis with the highest possible purity. The elements used in ZnSe growth were commercially obtained (Alpha Aesar-Puratronic) with purity of $6 \mathrm{~N}$ in the case of $Z n$ and $5 \mathrm{~N}$ in the case of Se. Both elements were separately distilled at least three times in dynamic vacuum to reduce impurities and to avoid the oxidized elements presence [8-14] (Figure 1). 


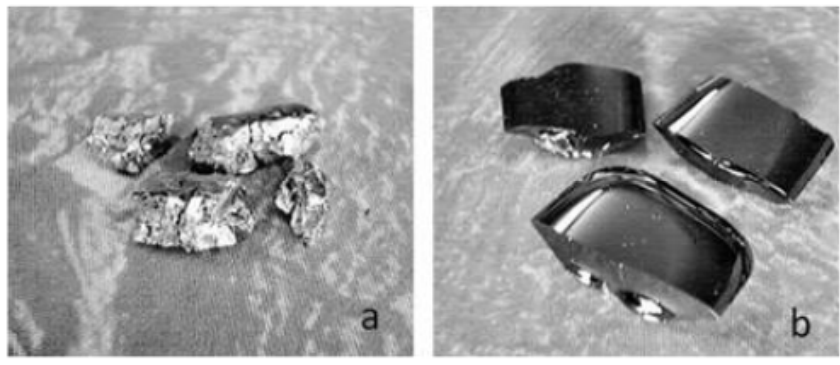

Figure 1: Purified elements by dynamic vacuum distillation. a) Zinc, b) Selenium.

Polycrystalline ZnSe was subsequently synthesized from $\mathrm{Zn}$ and Se stoichiometrically loaded in a quartz ampoule that was previously degassed in a vacuum line at a temperature of $1000{ }^{\circ} \mathrm{C}$ during $24 \mathrm{hs}$. The loaded ampoule was sealed under high vacuum $\left(10^{-6}\right.$ Torr). A flame, provided by a torch fuelled with methane and oxygen, heated the ampoule in order to produce the chemical reaction. The polycrystalline compound once obtained was grounded and placed in the closed end of a quartz tube. Quartz wool was set in the opened end of the tube to avoid pumps contamination by the crystalline powder. The tube was then placed inside the vacuum line with the opened end towards the pumps. The charge was heated above the $\mathrm{Zn}$ melting point to degas the elements that did not previously reacted so vapor pressure did not prevent the ingot growth [10].

Tubular furnaces were designed and built for single crystalline growth of II-VI semiconductor ingots. The employed technique was vapor phase transport with $\mathrm{I}_{2}$ as gaseous carrier. Different attempts were always carried out employing $3 \mathrm{~g}$ of polycrystalline $\mathrm{ZnSe}$ and changing the growth conditions: $I_{2}$ mass, growth time, furnace temperature profiles and ampoule temperature gradients in order to improve the crystalline quality [15, $16]$ to ensure repeatability.

Ampoules had a diameter of $15 \mathrm{~mm}$, a length of 17 $\mathrm{cm}$ and an angular conical aperture of $30^{\circ}$. The furnace temperature profile measurement allowed the temperature gradient calculation in the growth zone using the temperature values at the ampoule ends [15]. The ampoule maximum temperature always was 838 ${ }^{\circ} \mathrm{C}$ and different ampoule minimum temperatures have been tested to get the best single crystalline growth.

Wafers, $1 \mathrm{~mm}$ thick, were cut from the ingot with a wire saw (South Bay Technology) with no crystallographic orientation or in the (111) crystallographic orientation. This particular orientation was found in the grown ingot by $x$-ray diffraction (Laue technique) using the equipment Philips PW 3710. Wafers were mechanically polished (Minimet - Buehler) with $1 \mu \mathrm{m}$ Alpha Alumina Powder agglomerate free (Leco) to get soft and planar surfaces.

Reagents for chemical etching assessment were chosen according to the information found in the References [17-19]. At this stage samples observation was carried out by a metallographic microscope (Union Versamet 5279) (OM) that counts on a Nomarski interference contrast device and by scanning electronic microscopy (SEM) with an ESEM XL30. Dislocations density was calculated by counting the etch pits on the micrographs. The adjacent subgrains misorientation was computed using the Read-Shockley approximation $[20,21]$. The method applicability to this material was settled by references [12, 22]. Average values were obtained considering measurements all over the surface of the wafer.

The samples were slimmed in planar geometry using a precision ionic polishing system (PIPS), Model Gatan $691\left(\mathrm{~V}_{\mathrm{Ar}+}<4 \mathrm{kV}\right)$ for transmission electronic microscopy (TEM) examination. Samples observation from ingots grown in our lab were done with a JEOL JEM 2000 FX (W thermo-ionic filament) with an operation voltage of $200 \mathrm{kV}$. Low and high resolution micrographs were obtained by a Gatan (CCD) camera coupled to a JEOL JEM 3000 (field emission cannon) which employed an operating voltage of $300 \mathrm{kV}$. Meanwhile, the ZnSe commercial substrate was observed by a Philips CM200 UT with $\mathrm{LaB}_{6}$ filament that employed an operating voltage of $200 \mathrm{kV}$.

The possibility of defects presence in the micrometric or nanometric range determined the employment of different instruments with different potentialities.
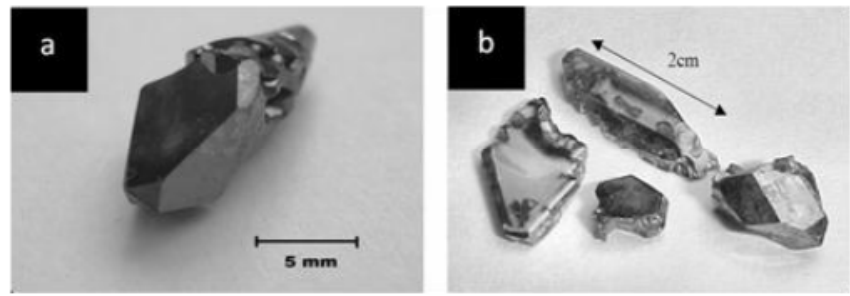

Figure 2: a) ZnSe ingot $(F)$ grown by chemical vapor phase transport with iodine as gaseous carrier b) Well defined crystallographic planes observed in different $\mathrm{ZnSe}$ single crystals. 


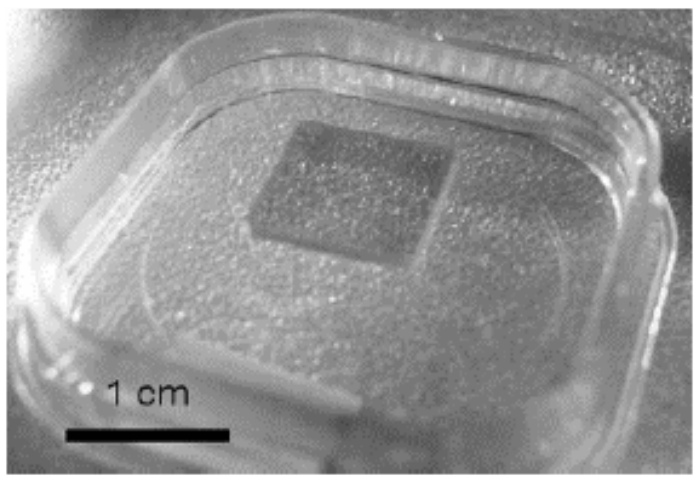

Figure 3: Commercial ZnSe wafer with (110) crystallographic orientation.

\section{RESULTS AND DISCUSSION}

Figure 2a shows the best ZnSe ingot $(F)$ grown by chemical transport with $\mathrm{I}_{2}$ as gaseous carrier, while in Figure $\mathbf{2 b}$ well defined crystallographic planes are observed in different $\mathrm{ZnSe}$ single crystals, all of them grown at our lab. Figure 3 shows the ZnSe commercial wafer with (110) crystallographic orientation.

A growth time of 22 days with a temperature gradient at the growth zone of $1.36\left({ }^{\circ} \mathrm{C} / \mathrm{cm}\right)$ were determined as the best single crystalline growth conditions. Change of the loaded iodine mass was taken into account through a parameter defined by the ratio of iodine mass and the ampoule free volume. The increment of the defined parameter determined better crystalline quality being $3.45\left(\mathrm{mg} / \mathrm{cm}^{3}\right)$ the best for the ZnSe grown ingot. This value is in the range of no iodine doping found by Yasutoshi Noda et al. [23].
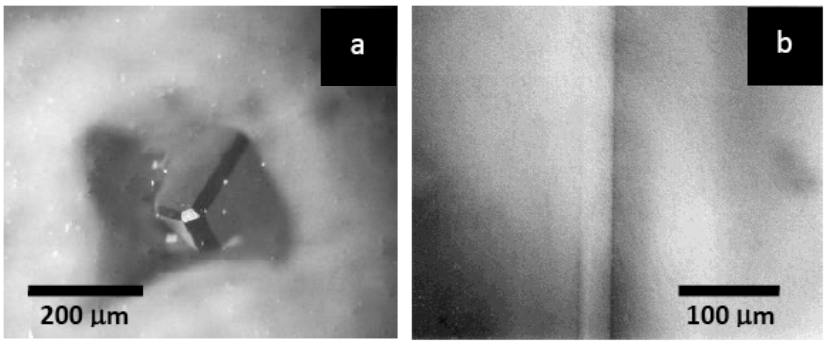

Figure 4: a) Occluded crystal in a lab grown ZnSe material, b) twin observed in other lab grown ZnSe material. Both wafers were cut from different ingots obtained out of the best growth conditions.

Ingots with macroscopic defects were discarded after these defects were studied. If the growth conditions were not appropriate, occluded crystals with well defined planar surfaces could be observed by optical microscopy (Figure 4a). Occluded crystals lack ensures greater useful volume available for devices manufacture. Also some twins (Figure 4b) could be observed in a different wafer showing that oxygen removal was not properly done [11] meanwhile ampoule charge. When growth conditions were accomplished then $\mathrm{ZnSe}$ single crystalline ingots were evenly yellowish and transparent (Figure 2) though ingot $(F)$ presented in this paper had the best mentioned properties. Figure $\mathbf{5}$ shows an occluded crystal in ZnSe commercial substrate, type of defect that was present with a very low density.

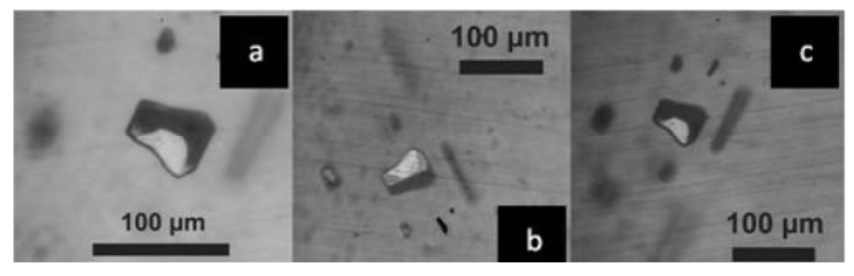

Figure 5: The same group of occluded crystals in the commercial ZnSe wafer was shown with different magnifications or different positions (5a, $5 \mathbf{b}$ and $\mathbf{5 c}$ ).
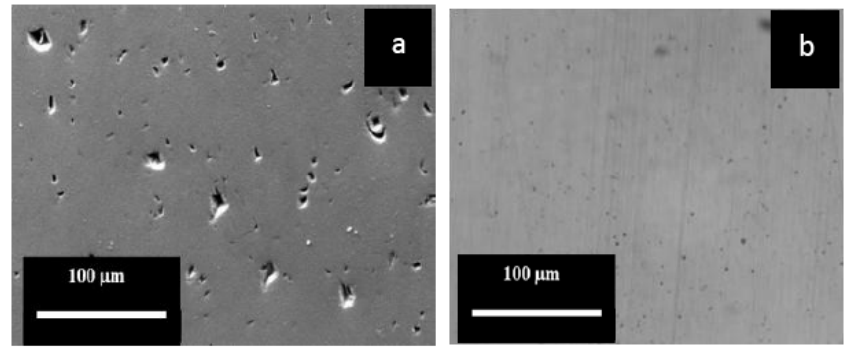

Figure 6: a) SEM micrograph of $\mathrm{F} 1$ wafer etched with the chemical solution $\mathrm{KMnO}_{4}+\mathrm{H}_{2} \mathrm{SO}_{4}$ b) OM micrograph of $\mathrm{ZnSe}$ commercial wafer etched with the chemical solution $\mathrm{KMnO}_{4}+\mathrm{H}_{2} \mathrm{SO}_{4}$.

Defects chemical etching in the samples had been carried out employing strong oxidizing reagents in acidic medium at room temperature. In every case strictly controlled time of the sample exposure to the corrosive media had been employed in order to obtain well defined etch pits over the sample surface. The presence of these corrosion figures corresponded to the existence of stressed areas whose origin was the presence of crystal defects such as dislocations, precipitates and agglomerates of vacancies or impurities. A massive attack to the sample surface by the reagent had to be avoided because the material might be over-etched and no valuable information could be obtained. References could be found in the literature using strongly corrosive media at elevated temperatures and for prolonged times [2, 5, 12, 24-26]. The experience of the authors of this paper was that under these conditions the wafer surface suffers widespread damage that did not provide valid information of the material crystalline quality. 
In Table 1 are shown the chemical etching solutions and also the average values of the dislocations density $\left\langle\delta_{\perp}\right\rangle$ and average misorientation between adjacent subgrains $\langle\phi\rangle$ obtained on the micrographs in every case of ingot $\mathrm{F}$. The digit, in the chemically etched wafer column, refers to the crystalline ingot cut number. Figure 6a shows a SEM micrograph corresponding to the wafer $\mathrm{F} 1$ chemically etched by $\mathrm{KMnO}_{4}+\mathrm{H}_{2} \mathrm{SO}_{4}$ solution.

Table 1: Chemical Etching Solutions, Average Dislocations Density and Average Adjacent Subgrains Misorientation in $\mathrm{ZnSe}$ Wafers from Ingot $F$

\begin{tabular}{|c|c|c|c|}
\hline Wafer & Chemical Solution & $\left\langle\delta_{\perp}>\left(\mathbf{c m}^{-2}\right)\right.$ & $<\phi>(")$ \\
\hline \hline $\mathrm{F} 1$ & $\mathrm{CrO}_{3}+\mathrm{Na}_{2} \mathrm{SO}_{4}$ & $210^{5}$ & 12 \\
\hline $\mathrm{F} 2$ & $\mathrm{CrO}_{3}+\mathrm{Na}_{2} \mathrm{SO}_{4}$ & $210^{5}$ & 12 \\
\hline $\mathrm{F} 1$ & $\mathrm{~K}_{2} \mathrm{Cr}_{2} \mathrm{O}_{7}+\mathrm{HCl}$ & $310^{5}$ & 14 \\
\hline $\mathrm{F} 2$ & $\mathrm{~K}_{2} \mathrm{Cr}_{2} \mathrm{O}_{7}+\mathrm{HCl}$ & $110^{5}$ & 10 \\
\hline $\mathrm{F} 1$ & $\mathrm{KMnO}_{4}+\mathrm{H}_{2} \mathrm{SO}_{4}$ & $610^{5}$ & 22 \\
\hline
\end{tabular}

Values of the same order for average dislocations density and average adjacent subgrains angular misorientation were obtained for all wafers $(F)$. Values were also of the same order to those obtained in wafers from all the other grown ingots in different conditions. Then, the effect of the reagent $\mathrm{KMnO}_{4}+\mathrm{H}_{2} \mathrm{SO}_{4}$ on the surface of the wafer with (111) crystallographic orientation has also been studied. Table 2 shows the measurement values for this plane employing this chemical solution that was recommended for chemical polishing by the bibliography [22].

Results showed that (111) plane did not present a major reactivity to this chemical solution since if it were employed on the same material, without selecting a preferential plane, the obtained values were of the same order. All dislocations density measurements have values of the same order which are also similar to those found in the References [22, 27, 28] showing that the crystalline quality of the lab grown semiconductor is appropriate for optical devices. In the case of $\mathrm{ZnSe}$ commercial substrate, the average dislocations density and average adjacent subgrains misorientation computed using optical micrographs as Figure $\mathbf{6 b}$, taken all over its surface, were $\left\langle\delta_{\perp}\right\rangle=510^{4} \mathrm{~cm}^{-2}$ and $\langle\phi\rangle=4$ ". So, dislocations density is lower in one order and adjacent subgrains misorientation is about three times less, both cases respect to the grown material in our laboratory. Although our grown material have a higher dislocations density and subgrains misorientation compared with this unique commercial analyzed wafer, these values are acceptable for manufacturing devices as they show a crystalline quality similar to those in the reported literature $[22,27-$ 29]. Otherwise larger $\mathrm{ZnSe}$ single crystals have been successfully obtained by the rotational chemical vapor transport (R-CVT) using iodine as a transport agent that were used for the fabrication of white LED's [26]. So, this growth method has to be taken care and improved.

Table 2: Chemical Etching on the (111) Crystallographic Plane

\begin{tabular}{|c|c|c|c|}
\hline Plane & Chemical Solution & $<\delta_{\perp}>\left(\mathbf{c m}^{-2}\right)$ & $<\phi>$ (") \\
\hline \hline$(111)$ & $\mathrm{KMnO}_{4}+\mathrm{H}_{2} \mathrm{SO}_{4}$ & $210^{5}$ & 14 \\
\hline
\end{tabular}

Figures $\mathbf{6 a}$ and $\mathbf{6 b}$ observation showed that $\mathrm{ZnSe}$ grown at our lab is more reactive than the commercial material as in both micrographs the same scale was used.

All wafers were studied by TEM and comparable micrographs were obtained. So, only $\mathrm{F} 1$ wafer results are shown. The assessment sequence of defects presence by chemical etching and TEM is due to the fact that the latter is a destructive technique.
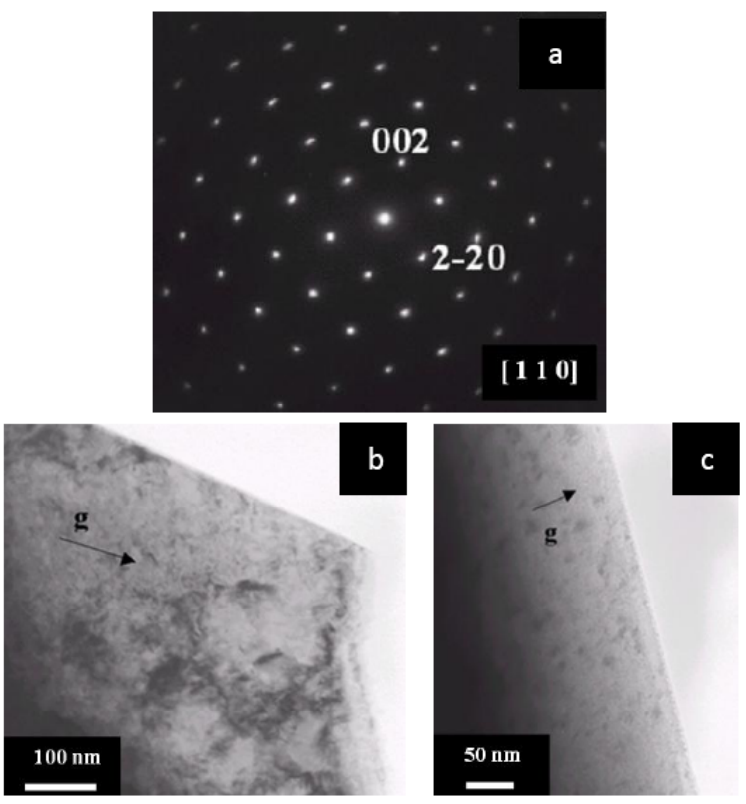

Figure 7: TEM observations on $\mathrm{ZnSe}$ from $\mathrm{F}$ ingot. a) Transmission electron diffraction (TED) diagram with [110] zone axis, b) TEM micrograph in the direction $\mathbf{g}=[1 \overline{1} 1], \mathbf{c})$ TEM micrograph in the direction $\mathbf{g}=[2 \overline{2} 0]$. 
Figure $\mathbf{7 a}$ is the transmission electron diffraction diagram with [110] zone axis. This plane was used to explore the existence of dislocations taking different $\mathbf{g}$ directions of the reciprocal space by means of the two beams technique. The micrographs obtained with $\mathbf{g}=$ $[1 \overline{1} 1]$ and $\mathbf{g}=[2 \overline{2} 0]$ did not show dislocations lines as can be respectively seen in Figures $\mathbf{7 b}$ and $\mathbf{7 c}$.
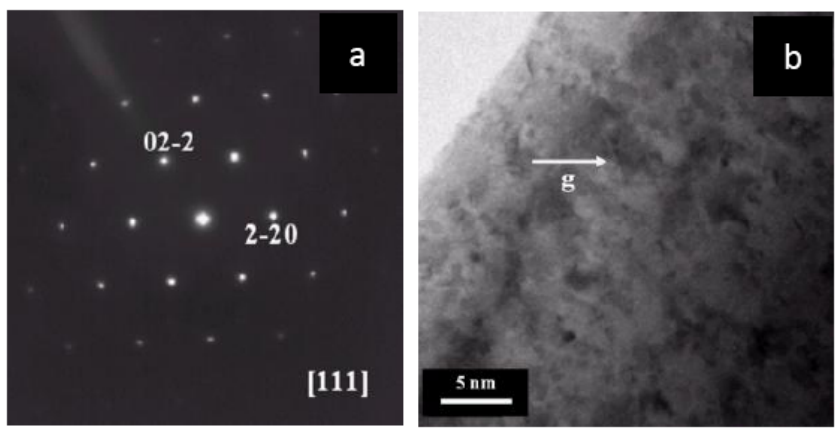

Figure 8: TEM observations on ZnSe F1 wafer. a) Transmission electron diffraction (TED) diagram with [111] zone axis. b) TEM Micrograph with $\mathbf{g}=[2 \overline{2} 0]$.

Dislocations presence was also analyzed by choosing the [111] zone axis and taking $\mathbf{g}=[2 \overline{2} 0]$ (Figures $\mathbf{8 a}$ and $\mathbf{8 b}$ ). These regions, observed by TEM, present neither lines nor loops of dislocations.

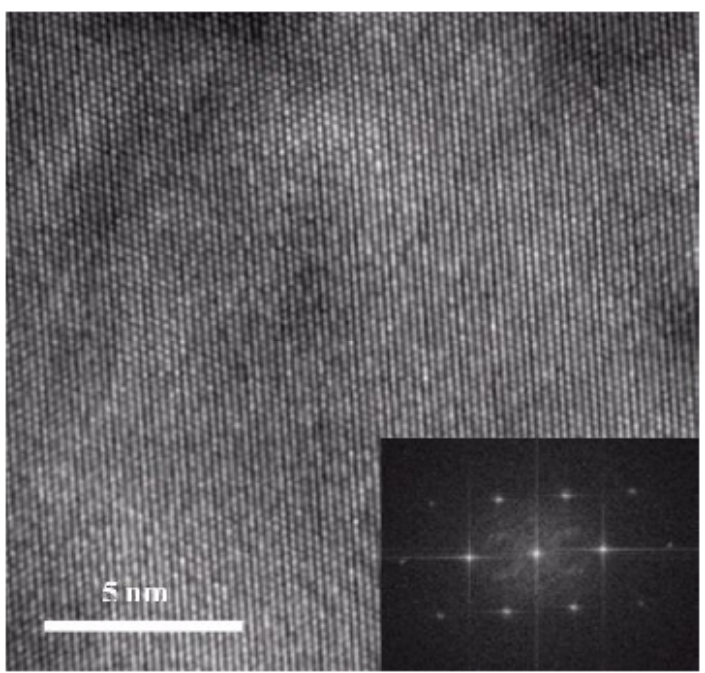

Figure 9 High resolution image (HRTEM) with [111] zone axis on $\mathrm{ZnSe} \mathrm{F1}$ wafer. Fourier transformed image is shown.

High resolution was used, in this case, to determine the existence of dislocations at local order, since the analysis with lower resolutions show a larger analyzed area. Then high resolution TEM images with different magnifications were obtained with different [111] zone axis. Fourier transformed image is inserted at the right bottom of Figure 9 . It corresponds to the above mentioned orientation and is identical to the TED diagram of Figure $\mathbf{8 a}$. Figure 10 shows an HRTEM image taken with the same zone axis and a major magnification than in the previous figure. None of these observations present dislocations at nanometric local level.

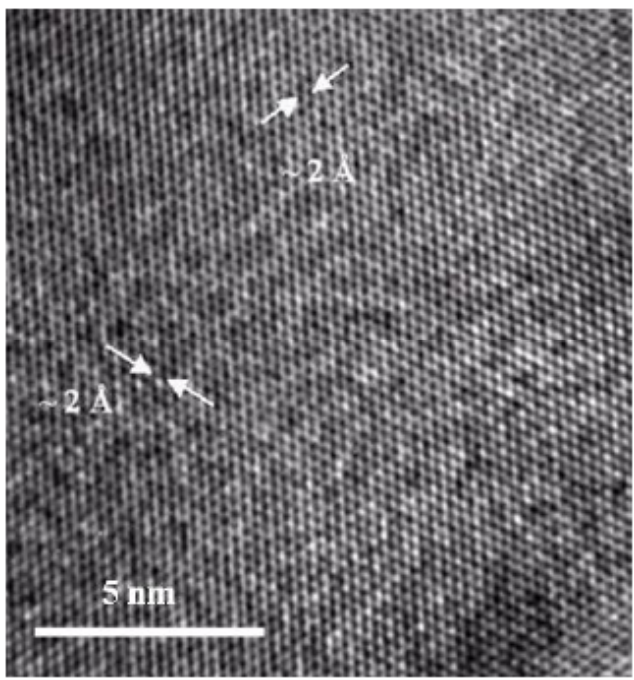

Figure 10: High resolution image (HRTEM) with [111] zone axis on $\mathrm{ZnSe} \mathrm{F} 1$ wafer.

TEM micrographs, in all cases, allowed observing an excellent structural order of the grown material: defect free zones that correspond to areas between contiguous dislocations on the micrographs as in Figure 6a.

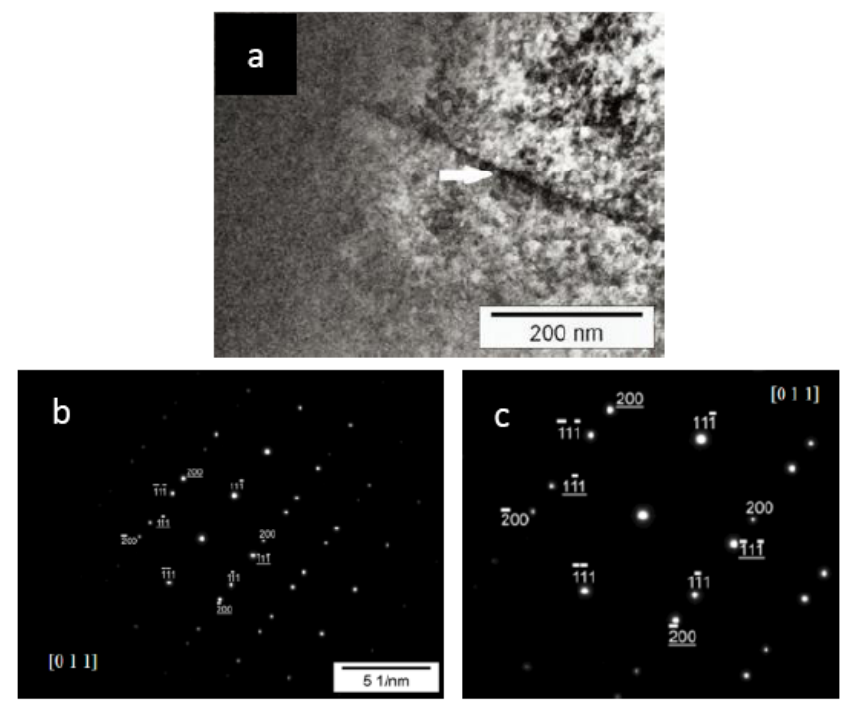

Figure 11: a) Twin on ZnSe commercial sample observed by TEM, b) TED diagram with [011] zone axis, c) magnification of TED (diagram $\mathbf{b}$ ) to observe twin planes indices.

However, in the case of commercial $\mathrm{ZnSe}$, it was observed a twin interface (Figure 11a) in which the electron diffraction diagram corresponds to the [011] 
zone axis (Figure 11b and 11c) common to the twin related crystals being the twin plane parallel to the common planes $(11 \overline{1})$ and ( $\overline{1} \overline{1} 1)$ although HRTEM micrographs showed excellent structural order (Figure 12a) between dislocations (Figure 6b)

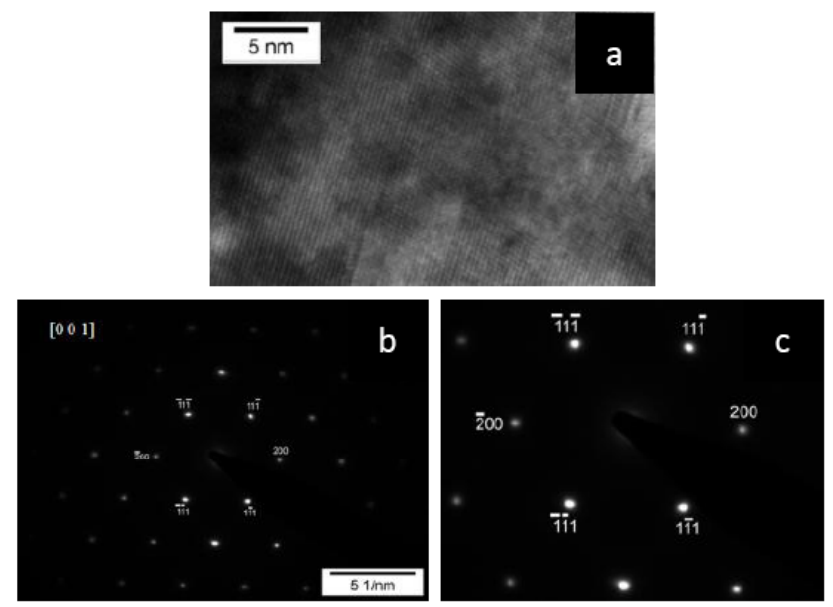

Figure 12: a) High resolution image (HRTEM) with [001] zone axis on ZnSe commercial sample, b) TED diagram with [001] zone axis, c) magnification of TED (diagram b) to observe indexation.

Dislocations or stacking faults were not observed in any of the TEM studied ZnSe samples. Stacking faults as well as high density pores were present in commercial ZnTe both II-VI semiconductors with FCC structure grown by HPB $[30,31]$.

\section{CONCLUSIONS}

Characterization values of single crystalline $\mathrm{ZnSe}$ grown by CVT, as well as by HPB allow us to conclude that:

- Macroscopic crystalline defects were observed by optical microscopy in the commercial $\mathrm{ZnSe}$ material as well as in the ZnSe material grown at the lab by CVT but only, in the latter case, when growth was not in optimal conditions

- Linear defects characterization could be done because of the employment of two complementary techniques: TEM and chemical etching.

- $\quad$ TEM observations determined that dislocations did not exist in the micrometric or nanometric range in our grown lab single crystalline material but a twin could be observed in the commercial material. It might be generated by the HPB growth rate employed.
- $\quad$ Chemical etching method was used to determine dislocations density and contiguous subgrains misorientation.

- Transmission electron microscopy (TEM) micrographs determined an excellent structural order between dislocations.

- Different chemical etching solutions could be used for linear defects detection independently of the etching crystallographic plane.

- ZnSe (111) crystallographic plane did not present major sensitivity for the chemical solution $\mathrm{KMnO}_{4}+\mathrm{H}_{2} \mathrm{SO}_{4}$ since similar values were obtained in $\left\langle\delta_{\perp}\right\rangle$ and $\langle\phi\rangle$ employing this reagent without selecting an etching preferential crystallographic plane.

- Dislocations density and misorientation between adjacent subgrains were comparable to the values found in literature which confirm the suitable crystalline quality of our material for its employment in optoelectronics devices.

- $\quad$ CVT optimal growth conditions have achieved to get single crystals in a controlled and repetitive way.

\section{ACKNOWLEDGEMENTS}

The authors are grateful to Professor Miguel Á. Alario Franco (Inorganic Chemistry Department, UCMSpain) for the authorization of the use of the transmission electronic microscopes facilities. Also the authors are grateful to the Institutions CONICET and FRBA-UTN for the grants (respectively PIP 5468-05 and $25 / C 116$ ) that allowed us to achieve this project.

\section{REFERENCES}

[1] Yoneta M, Nanami K, Uechi H, Ohishi M, Saito H, Yoshino K Epitaxial growth and characterization of $\mathrm{Cl}$ doped $\mathrm{ZnSe}$ layer by MBE; J Cryst Growth 2002; 237-239: 1545-1549. http://dx.doi.org/10.1016/S0022-0248(01)02365-X

[2] Li H, Jie W. Growth and characterization of bulk ZnSe single crystal by chemical vapour transport, J Cryst Growth 2003; 257: $110-115$.

http://dx.doi.org/10.1016/S0022-0248(03)01431-3

[3] Fujiwara S, Namikawa Y, Irikura M, Matsumoto K, Kotani T. Growth of dislocation-free ZnSe single crystal by CVT method, J Cryst Growth 2000; 219: 353-360. http://dx.doi.org/10.1016/S0022-0248(00)00671-0

[4] Li H, Gu Z, Zang H, Li W. Thermodynamic analysis and growth of ZnSe single crystals in Zn-Se-12 system, J Cryst Growth 2015; 415: 158-165.

http://dx.doi.org/10.1016/j.jcrysgro.2014.09.002 
[5] Yamauchi T, Takahara Y, Naitoh M, Narita N. Growth mechanism of ZnSe single crystal by chemical vapour transport method, Physica B 2006; 778: 376-377. http://dx.doi.org/10.1016/i.physb.2005.12.195

[6] Kannappan P, Dhanasekaran R. Studies on structural and optical properties of ZnSe and ZnSSe single crystals grown by CVT method, J Cryst Growth 2014; 401: 691-696. http://dx.doi.org/10.1016/j.jcrysgro.2013.11.030

[7] Fujita S, Yodo T, Sasaki A. Lattice-mismatch effects on properties in ZnSe layer grown on GaAs substrate by low pressure OMVPE, J Cryst Growth 1985; 72(1-2): 27-30. http://dx.doi.org/10.1016/0022-0248(85)90113-7

[8] Shelpakova IR, Kosyakov VI, Kovalevski SV, Shestakov VA. The Use of Evaporation in Vacuum for Purification and Analysis of Zinc, Mater Res Bull 1998; 33(2): 173-181. http://dx.doi.org/10.1016/S0025-5408(97)00223-7

[9] Chulzhanovi YA, Nenashevi BG, Popov SP, Chulzhanova MG. Preparation of High-Purity Chalcogens, Chemistry for Sustainable Development 2000; 8: 29-31.

[10] Heredia E, Trigubó AB, Gilabert U, Cánepa H, D'Elía R, Marrero J, et al. Purificación del Cd por destilación en vacío dinámico, Superficies y Vacío 2000; 10: 28-31.

[11] Mycielski A, Szadkowski AJ, Kowalczyk L, Zielínski M, Lusakowska E, Witkowska B, et al. Technology and Characterisation of Single-Crystalline Substrates made of ZnSe-Based Wide-gap II-VI Semiconductor Compounds, Phys Stat Sol (b) 2002; 229(1): 189-192. http://dx.doi.org/10.1002/1521-3951(200201)229:1<189::AIDPSSB189>3.0.CO;2-0

[12] Cantwell G, Harsh WC, Cotal HL, Markey BG, McKeever SWS, Thomas JE. Growth and characterization of substrate-quality $\mathrm{ZnSe}$ single crystals using seeded physical vapor transport, J Appl Phys 1992; 71(6): 2931-2936. http://dx.doi.org/10.1063/1.351026

[13] Ali ST, Prasad DS, Munirathnam NR, Prakash TL. Purification of tellurium by single-run multiple vacuum distillation technique, Sep Purif Technol 2005; 43: 263-267. http://dx.doi.org/10.1016/j.seppur.2004.11.008

[14] Kovalevsky SV, Shelpakova IR. High-Purity zinc, cadmium, tellurium, indium and Gallium: preparation and analysis, Chemistry for Sustainable Development 2000; 8: 85-87.

[15] Piper WW, Polish SJ. Vapor-Phase Growth of Single Crystals of II-VI Compounds, J Appl Phys 1961; 32: 12781279.

http://dx.doi.org/10.1063/1.1736219

[16] Mycielski A, Lusakowska E, Szadkowski A, Kowalcyk L. Low defect density, substrate quality crystals of the wide-gap II-VI compounds, obtained by physical vapour transport technique (PVT), J Cryst Growth 1998; 184-185: 1044-1047.

[17] Aliya D, in: Handbook ASM, edited by Vander Voort GF. Metallography and Microstructures, Vol. 9, ASM International, Materials Park, 2004, chap. 2.
[18] Trigubó AB, Di Stefano MC, D'Elía R, Cánepa H, Heredia E, Aguirre $\mathrm{MH}$. Calidad cristalina del $\mathrm{ZnSe}$ obtenido por transporte químico con 12 como portador gaseoso, Superficies y Vacío 2007; 20(3): 21-25.

[19] Nuez García J, Geraci A, Tolley A, Di Stefano MC Cabanillas E, Martínez AM, D’Elía R, Heredia E, Trigubó A B. Estudio de la Morfología y Calidad Cristalina de ZnSe Monocristalino. Proceedings of the Congress SAM CONAMET 2013 held at Puerto Iguazú, Misiones, Argentina, August 20-23 2013. [CD-ROM. ISBN 978-950-579-276-4].

[20] Sangwall K. Etching of Crystals. Theory, Experiment and Application. Elsevier North-Holland, Amsterdam, 1987, p. 20.

[21] Read WT. Dislocations in crystals, Mc-Graw-Hill, New York, 1953, p. 156

[22] Ebina A, Asano K, Takahashi T. Crystallographic polarity and etching behaviour of Zn Se, Jap. J Appl Phys 1977; 16(9): 1563-1570.

http://dx.doi.org/10.1143/JJAP.16.1563

[23] Noda $Y$, Yonekura H, Okunuki $Y$, Nakazawa S. Crystal Growth of ZnSe in Closed Tube by Chemical Transport with lodine, Materials Transactions JIM 1995; 36(8): 1067-1073.

[24] Sankar N, Ramachandran K, Sanjeeviraja C. Growth and characterization of $\mathrm{ZnSe}$ and phosphorus-doped $\mathrm{ZnSe}$ single crystals, J Cryst Growth 2002; 235: 195-200. http://dx.doi.org/10.1016/S0022-0248(01)02044-9

[25] Fang C, Gu Q, Wei J, Pan Q, Shin W, Wang J. Growth of ZnSe single crystals, J Cryst Growth 2000; 209: 542-546. http://dx.doi.org/10.1016/S0022-0248(99)00616-8

[26] Fujiwara S, Namikawa Y, Nakamura T, Tatsumi M. Growth of large ZnSe single crystal by R-CVT method, J Cryst Growth 2005; 275: e415-e419 http://dx.doi.org/10.1016/j.jcrysgro.2004.11.012

[27] Maruyama K, Suto K, Nishizawa J. ZnSe horizontal traveling solvent growth using selenium solution, J Cryst Growth 2000; 216(1-4): 113-118

http://dx.doi.org/10.1016/S0022-0248(00)00361-4

[28] Wang JF, Omino A, Isshiki M. Melt growth of twin-free ZnSe single crystals, J Cryst Growth 2000; 214-215(1-4): 875-879. http://dx.doi.org/10.1016/S0022-0248(00)00219-0

[29] Shkir M, Vijayan N, Nasir M, Wahab MA, Bhagavannarayana G. Characterization of ZnSe single crystal grown by VBT using two zone tubular furnace: An excellent material for optoelectronic devices, Optik 2013; 124: 985-989. http://dx.doi.org/10.1016/j.ijleo.2013.01.026

[30] Trigubó AB, Di Stefano MC, Gilabert U, Martínez AM, D'Elía $\mathrm{R}$, Cánepa $\mathrm{H}$, et al. TEM, Chemical Etching and FTIR Characterization of ZnTe Grown by Physical Vapor Transport, Cryst Res Technol 2010; 45(8): 817-824. http://dx.doi.org/10.1002/crat.200900297

[31] Trigubó AB, D`Elía R, Heredia E. (private communication).

http://dx.doi.org/10.15379/2408-977X.2015.02.02.5

(c) 2015 D’Elía et al.; Licensee Cosmos Scholars Publishing House.

This is an open access article licensed under the terms of the Creative Commons Attribution Non-Commercial License

(http://creativecommons.org/licenses/by-nc/3.0/), which permits unrestricted, non-commercial use, distribution and reproduction in any medium, provided the work is properly cited. 